\title{
Daily Emotion in Non-Suicidal Self-Injury
}

\section{Sarah Elizabeth Victor and E. David Klonsky}

\section{University of British Columbia}

Objective: While major theories of non-suicidal self-injury (NSSI) emphasize the behavior's role in emotion regulation, little is known about the daily emotional experiences of self-injurers. This study investigated the specific emotions that are characteristic of those who engage in NSSI. Method: University students $(n=84)$ with either no history or a recent history of NSSI completed daily diary and retrospective measures of emotional experience. To evaluate generalizability of findings, the retrospective measure was also administered to a diverse sample of U.S. adults $(n=92)$ with and without histories of NSSI. Results: Results indicate that self-injurers experience greater negative emotionality, particularly self-dissatisfaction, compared to individuals with no NSSI history. Self-injurers also reported less positive emotion, but these effects were smaller. The pattern of results was similar when controlling for Axis I psychopathology and borderline personality disorder symptoms. Conclusions: Individuals who engage in NSSI experience more negative emotions, generally, and more self-dissatisfaction, specifically. Findings contribute to the growing literature on the role of emotion in the etiology and functions of NSSI. (C) 2013 Wiley Periodicals, Inc. J. Clin. Psychol. 00:1-12, 2013.

Keywords: self-injury; self-harm; emotion; diary; self-derogation

Non-suicidal self-injury (NSSI) is the deliberate, self-inflicted injury to one's body that results in tissue damage, is not socially sanctioned, and is not suicidal in nature (International Society for the Study of Self-Injury, 2007). These behaviors, such as cutting, burning, and self-hitting, are prevalent in a variety of populations, with approximately $6 \%$ of adults (Klonsky, 2011) and 15\% of adolescents (Laye-Gindhu \& Schonert-Reichl, 2005) having engaged in NSSI. In addition to the risk of physical harm inherent in NSSI, these behaviors are associated with a variety of types of psychopathology, such as mood, anxiety, and eating disorders, as well as risk of suicide ideation and attempts (Claes, Klonsky, Muehlenkamp, Kuppens, \& Vandereycken, 2010; Klonsky, May, \& Glenn, 2012; Nock, Joiner, Gordon, Lloyd-Richardson, \& Prinstein, 2006).

\section{NSSI and Emotional Experiences}

Many researchers have concluded that NSSI is closely related to emotional experience. The experiential avoidance model of NSSI suggests that these behaviors are primarily reinforced through reduction or removal of aversive emotional experiences (Chapman, Gratz, \& Brown, 2006; Nock \& Prinstein, 2005). This model is consistent with an empirical review by Klonsky (2007) indicating that the most frequent motivation for NSSI is to regulate negative emotional experiences. NSSI is typically preceded by high arousal negative emotions (Claes et al., 2010), and engaging in NSSI is associated with large decreases in these emotions (Kleindienst et al., 2008) along with increases in emotions such as calm and relief (Claes et al., 2010; Klonsky, 2009). This pattern of results has also been found in studies using ecological momentary assessment methodology (Nock, Prinstein, \& Sterba, 2009).

\section{NSSI and Depression}

NSSI is consistently associated with depressive symptoms and the presence of mood disorders. A history of NSSI has been associated with elevated self-reported depression among

Please address correspondence to: Sarah Victor, University of British Columbia, 2136 West Mall, Vancouver, BC, Canada V6T1Z4. E-mail: sevictor@psych.ubc.ca 
treatment-seeking and community samples of adults (Klonsky, Oltmanns, \& Turkheimer, 2003; Selby, Bender, Gordon, Nock, \& Joiner, 2012). Additionally, self-injurers are more likely to be diagnosed with a mood disorder, such as major depressive disorder, than individuals with no history of NSSI (Selby et al., 2012). Sadness and depression frequently occur immediately preceding and following NSSI (Kamphuis, Ruyling, \& Reijntjes, 2007; Klonsky, 2009).

\section{NSSI and Anxiety}

NSSI is associated with self-reported anxiety (Klonsky et al., 2003; Selby et al., 2012), and anxiety frequently precedes engagement in NSSI (Chapman \& Dixon-Gordon, 2007). While some research suggests that adults with a history of NSSI are more likely to be diagnosed with an anxiety disorder (Chartrand, Sareen, Toews, \& Bolton, 2011), this finding has not always been replicated (Selby et al., 2012).

\section{NSSI and Self-Directed Negative Emotions}

Studies of community adolescents have found that those with a history of NSSI report lower global self-worth and self-esteem (Claes et al., 2010), as well as elevated levels of negative selfevaluation compared to adolescents with no history of NSSI (Laye-Gindhu \& Schonert-Reichl, 2005). Self-directed negative emotions are also elevated among adults with NSSI, including self-directed anger, self-derogation, and low self-esteem (Klonsky et al., 2003; St. Germain \& Hooley, 2012). Notably, studies have shown that self-criticism is most strongly related to NSSI as compared to indirect forms of self-injury such as substance abuse and disordered eating behavior, suggesting a unique and potentially important relationship between self-criticism and NSSI (St. Germain \& Hooley, 2012). The consistent relationships of self-derogation, self-criticism, and low self-esteem to NSSI are particularly noteworthy given the frequent use of NSSI as a form of self-punishment (see Klonsky, 2007, for review).

\section{Limitations in Existing Research on Emotions and NSSI}

Several limitations restrict the contributions of existing research to our understanding of emotion in NSSI. First, much research has assessed broad constructs relevant to emotion, such as emotion-related psychopathologies (e.g., depression, anxiety), or constructs, such as emotion regulation (Gratz \& Tull, 2010; Nock et al., 2006), without assessing emotional experience directly. Second, research examining emotion more directly has typically relied on retrospective measures of emotional experience. Because individuals who engage in NSSI may have difficulty naming and expressing emotions (Zlotnick et al., 1996), it is especially important to use measurement tools that minimize additional sources of error, such as recall bias. Third, those studies that do use more ecologically valid methods of emotional assessment primarily focus on the emotions that immediately precede and follow NSSI behaviors (e.g., Nock et al., 2009). This research is useful for examining the role of emotion in precipitating or reinforcing instances of NSSI. However, it is also important to examine emotions that characterize the daily lives of individuals who self-injure, and how these emotional experiences differ between those who do and do not choose NSSI as a coping method-an aim not yet addressed through ecologically valid measures. Fourth, research has not yet examined specific emotional experiences among self-injurers in a way that incorporates multiple dimensions (e.g., frequency, intensity, and duration of each emotion) into the assessment of specific emotional states. Finally, researchers have yet to investigate how emotional experiences related to NSSI may differ by the type of population being investigated (e.g., by clinical status or age).

The present research was designed to address these limitations. Given the lack of clear theoretical or empirical work suggesting specific hypotheses, these analyses were exploratory in nature. We administered both daily diary and retrospective measures of emotional experience to university students with and without a history of NSSI. These measures included indices of emotional reactivity dimensions (frequency, intensity, and persistence) that were used to calculate scores for 20 specific emotions. In addition, the retrospective measure was administered to 
a community sample of adults to assess the generalizability of relationships between emotional experience and NSSI across nonclinical populations that vary by age, educational attainment, ethnicity, and country of residence.

\section{Study 1: Undergraduate Diary Study Sample}

\section{Recruitment and Procedures}

Participants in study 1 were recruited from the student population of a large public Canadian university and its surrounding community for either a history of NSSI in the past 6 months or no history of lifetime NSSI. To focus on the most clinically relevant NSSI behaviors, participants in the recent NSSI group had to endorse a history of at least one behavior resulting in tissue damage (e.g., cutting, burning, or carving); individuals endorsing only less severe behaviors (e.g., skin-picking or self-hitting) were excluded. Potential participants were excluded if they were unable to provide informed consent due to cognitive impairment or lack of English fluency. Study recruitment and procedures were conducted in accordance with the behavioral research ethics board at the university. Participants in the study received an honorarium or course credit for participation.

Participants completed baseline measures during a laboratory-based session, during which they reported on aspects of their emotional, psychiatric, and behavioral experiences, including their history of NSSI. A total of 84 participants completed this portion of the study protocol; 57 had no lifetime history of any NSSI, while 27 had engaged in NSSI with tissue damage in the past 6 months.

Participants were invited to complete a diary once a day for 14 days following their session. This diary included measures of emotional experience and NSSI. Fifty-seven participants agreed to complete the daily diary; three participants subsequently chose to end participation in the diary, resulting in data from 54 participants. Of these participants, 36 participants had no history of NSSI, while 18 participants had a recent history of NSSI.

The daily diary measures took approximately five minutes per day to complete, and were completed as close to the end of each day as possible. Because of concerns regarding ease of administration, as well as research suggesting equivalence between paper and pencil and electronic diaries (Green, Rafaeli, Bolger, Shrout, \& Reis, 2006), the diary was completed using an electronic data collection system participants accessed from home. While a majority of participants completed the daily diary using an online survey service, participants were also provided with the option to complete the diary in paper format; two participants selected this option for some or all of their diary entries.

\section{Diary Measures}

NSSI. Participants were asked to report the number of times over the course of the day that they engaged in twelve NSSI behaviors (e.g., cutting, burning, and hitting), with the option to enter an "other NSSI" frequency. The behaviors assessed were drawn from the Inventory of Statements About Self-Injury (ISAS; see below for psychometric information).

Emotional experiences. Participants were asked to complete a measure of 20 specific emotions (10 positive, 10 negative) that they may have experienced over the course of each day. For each emotion, participants were asked to make three ratings indicating the emotion's frequency, intensity, and duration for that day. They rated each domain on a 5-point Likert scale. Lower scores indicated less frequent, less intense, and shorter-lasting emotions. Intensity and duration scores were only made if a participant's rating indicated that the emotion had occurred on that day.

While participants typically completed a majority of diary entries (median $=11$, mean $[M]=10.8$, standard deviation $[S D]=2.9$ ), there was a nontrivial amount of missing data: $23 \%$ of expected days' entries were missing. These rates are similar to other studies using 
undergraduate populations with elevated levels of psychopathology (Cummings et al., 2013; Moore, Elkins, McNulty, Kivisto, \& Handsel, 2011). A total of 38\% of expected emotion entries were missing. This was in part because of some participants failing to complete a diary entry that they had begun, and in part because an emotion's intensity and duration ratings were considered missing for days in which the participant did not experience that emotion. To address this issue, multiple data imputation was conducted. Values for missing data were estimated using linear regression across 20 sequential imputations. Following these imputations, specific emotion scores were calculated by averaging the frequency, intensity, and duration scores for each emotion to create an overall measure of each specific emotional state for each day (e.g., for each participant, the composite sad frequency, composite sad intensity, and composite sad duration were averaged to create a Sad score). Statistical tests were conducted on each of the imputed datasets separately, and then combined into a pooled estimation of between-group differences. Multiple imputations and pooled estimations were conducted in SPSS version 17 (IBM, 2009).

\section{Retrospective Measures}

NSSI. The ISAS (Klonsky \& Olino, 2008) assesses lifetime frequency of twelve methods of NSSI, as well as an "other" category. In addition to these items, the ISAS includes questions to determine the date of onset and offset of NSSI, to ascertain whether NSSI behaviors occurred in the last 6 months. The ISAS has high internal consistency as well as convergent validity and good test-retest reliability (Glenn \& Klonsky, 2011).

Demographics. To assess demographic variables, participants completed a background questionnaire developed by the research team. This measure includes assessment of gender, sexual orientation, and ethnic background.

Emotional experiences. The principal self-report measure used to assess emotional experience was a parallel measure to the emotion items used during the daily diary. Participants were asked to report their typical, rather than daily, frequency, intensity, and duration for the same 20 specific emotional experiences. Scores for 20 specific emotions were calculated in the same manner as the daily diary reports, except without the need to average scores over multiple assessment points.

Borderline personality disorder (BPD). Because NSSI and emotional difficulties are both hallmarks of BPD, symptoms of BPD were assessed through the McLean Screening Instrument for BPD (MSI-BPD; Zanarini et al., 2003). This 10-item self-report measure asks respondents to indicate their lifetime history of each of the nine BPD symptoms described in the Diagnostic and Statistical Manual of Mental Disorders Fourth Edition Text Revision (DSM-IV-TR). This measure has good sensitivity and specificity in identifying BPD cases, as determined via diagnostic interview (Zanarini et al., 2003).

Depression and anxiety. The Depression Anxiety Stress Scales (DASS-21; Lovibond, S. \& Lovibond, P., 1995) is a 21 -item self-report measure used to assess emotional and physical symptoms of depression, anxiety, and stress in the preceding week. This measure has demonstrated strong internal consistency and convergent validity, and shows greater ability to distinguish between depression and anxiety than other instruments (Antony, Bieling, Cox, Enns, \& Swinson, 1998; Lovibond , P., \& Lovibond, S. 1995).

Psychiatric diagnoses. In addition to the questionnaire measures described above, the first author conducted two interviews to assess for past and present psychopathology. The Structured Clinical Interview for DSM-IV Disorders (SCID-I/P; First, Spitzer, Gibbon, \& Williams, 2002) was used to assess Axis I psychopathology, including mood, anxiety, substance use, and eating disorders. The SCID-I/P shows fair to excellent inter-rater reliability and generally fair to excellent test-retest reliability (Zanarini et al., 2000). 
The BPD items from the Structured Interview for DSM-IV Personality (SIDP-IV; Pfohl, Blum, \& Zimmerman, 1997) were utilized to assess each of the nine DSM-IV symptoms of BPD. The SIDP-IV has demonstrated good reliability and validity (Rogers, 2001).

\section{Results}

Sociodemographics. Of the 84 participants included in these analyses, $60(71.4 \%)$ were female. Participants' ages ranged from 19 to 43 years, with a mean of $23.3(S D=5.1)$. A majority identified their ethnicity as East Asian $(n=46,54.8 \%)$; other ethnic backgrounds in the sample were Caucasian $(n=24,28.6 \%)$, South Asian $(n=5,6 \%)$, other or bi/multiracial $(n=5,6 \%)$, Hispanic $(n=2,2.4 \%)$, Middle Eastern $(n=1,1.2 \%)$, and African $(n=1,1.2 \%)$. None of the demographic characteristics were related to NSSI history or to participation in the diary component of the study.

Clinical characteristics. Complete data regarding clinical characteristics are presented in Table 1. Consistent with existing literature, self-injurers exhibited more psychopathology than those without. Individuals with NSSI met criteria for more Axis I diagnoses as assessed by the SCID-I/P, and were significantly more likely to have a diagnosis of an anxiety, mood, or eating disorder; substance use disorders were marginally more likely in the recent NSSI group $(p=.053)$. NSSI was associated with elevated depression, anxiety, and stress scale scores of the DASS-21, as well as with more BPD symptoms (excluding the NSSI symptom) on the SIDP and the MSI-BPD.

Among the participants with a history of NSSI, the median number of lifetime acts of NSSI was 144. The mean number of methods of NSSI was $6.9(S D=2.6)$, and all participants reported more than one method. In addition to endorsing a history of cutting $(85.2 \%)$, burning $(37 \%)$, and carving (33.3\%), all participants endorsed engaging in other, less severe types of NSSI. Lifetime frequency and number of methods of NSSI did not differ between those who did and did not complete the diary portion of the study. Complete descriptive data on NSSI history is presented in Table 2.

Daily specific emotions in NSSI. There were group differences for seven negative emotions (dissatisfied with self, ashamed, sad, anxious, lonely, nervous, and disgusted, all higher in those with NSSI) and for three positive emotions (joyful, cheerful, and happy, all lower in those with NSSI). The largest group differences were for dissatisfied with self, ashamed, and sad. Detailed results for these specific emotions are reported in Table 3.

Using partial correlations, we investigated how specific emotions were related to NSSI history after accounting for clinical and demographic characteristics. While multiple imputation does not permit the calculation of an exact pooled $p$ value for partial correlations, we were able to compare the relative size of each relationship based on $r$ values. After controlling for Axis I psychopathology (DASS subscale scores and number of SCID diagnoses), the largest relationships with NSSI history were shown for disgust $(r=.30)$ and cheerful $(r=-.29)$. After controlling for BPD symptoms (MSI-BPD and SIDP scores), the largest relationships were for disgust $(r=.24)$, cheerful $(r=-.27)$, and joy $(r=-.26)$. The pattern of results after controlling for gender was almost identical to the results without inclusion of any covariates. Detailed results regarding the relationship between NSSI history and all 20 specific emotions after controlling for clinical covariates are available from the corresponding author.

Retrospective specific emotions in NSSI. Of the 20 specific emotions assessed, between group differences were found for six positive emotions (happy, cheerful, enthusiastic, joyful, proud, and relaxed, all higher in the no NSSI group) and all 10 negative emotions (sad, nervous, irritable, afraid, angry, ashamed, disgusted, anxious, lonely, and dissatisfied with self, all higher in the NSSI group). As in the diary portion of the study, the largest group difference was selfdissatisfaction (Cohen's $d=1.35$ ). Detailed results for these emotions are reported in Table 3 . Self-dissatisfaction, as well as disgust, remained statistically significantly related to NSSI history after controlling for Axis I psychopathology and BPD symptoms, while most other specific 


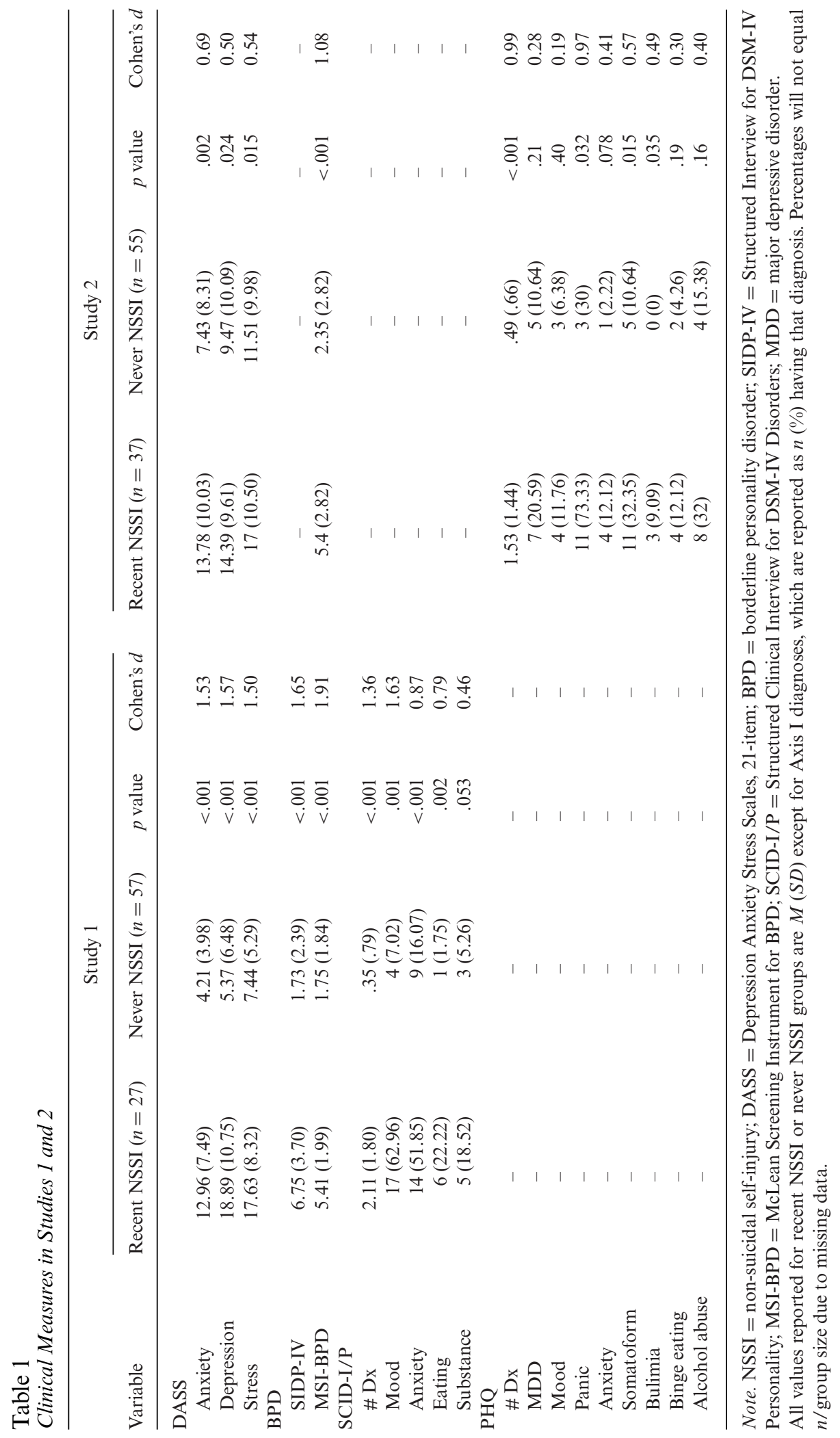


Table 2

Descriptive Data on Lifetime NSSI in Studies 1 and 2

\begin{tabular}{lcr}
\hline Variable & Study 1 & Study 2 \\
\hline NSSI Frequency (median) & 144 & 175 \\
Age of NSSI onset & $12.59(4.73)$ & $13.28(5.37)$ \\
Number of NSSI methods & $6.85(2.64)$ & $6.14(3.13)$ \\
Individual NSSI methods & & \\
Interfering with wound healing & $21(77.8)$ & $29(78.4)$ \\
Severe pinching & $20(74.1)$ & $22(59.5)$ \\
Banging & $20(74.1)$ & $18(48.6)$ \\
Hairpulling & $17(63)$ & $20(54.1)$ \\
Biting & $15(55.6)$ & $20(54.1)$ \\
Severe scratching & $15(55.6)$ & $20(54.1)$ \\
Cutting & $23(85.2)$ & $28(75.7)$ \\
Other & $8(29.6)$ & $5(13.5)$ \\
Rubbing with rough surfaces & $9(33.3)$ & $12(32.4)$ \\
Substances & $9(33.3)$ & $8(21.6)$ \\
Burning & $10(37)$ & $22(59.5)$ \\
Carving & $9(33.3)$ & $12(32.4)$ \\
Needles & $9(33.3)$ & $13(35.1)$ \\
\hline
\end{tabular}

Note. NSSI = non-suicidal self-injury.

Specific methods are listed with $n(\%)$; all other values are listed with $M(S D)$.

emotions were not robust to the inclusion of clinical covariates. Controlling for gender did not influence the statistical significance of any findings or the overall pattern of results.

\section{Study 2: Adult Community Sample}

\section{Recruitment and Procedures}

Participants in study 2 were adults in the United States recruited from a popular survey website (Amazon's Mechanical Turk, http://www.mechanicalturk.com; Buhrmester, Kwang, \& Gosling, 2011) on which individuals can participate in research for compensation. The advertisement for the study sought adults with a history of recent NSSI (in the past 6 months) or no history of NSSI. Study recruitment and procedures were conducted in accordance with the behavioral research ethics board at the authors' primary institution, and participants in the study received an honorarium for participation. Participants completed self-report measures using an online survey service that transmitted de-identified data to the research team. Because of the structure of Mechanical Turk and the nature of the data collected, participants were not invited to participate in a daily diary study. Participants completed a variety of self-report measures assessing NSSI, personality, emotional experiences, and psychopathology. A total of 92 participants completed the study protocol; 55 had no lifetime history of any NSSI, while 37 had engaged in NSSI with tissue damage in the past 6 months.

\section{Measures}

The measures for NSSI, demographics, emotional experiences, BPD, depression, and anxiety are the same as study 1 .

Psychiatric symptoms. To assess for the presence of Axis I psychopathology, participants completed the Patient Health Questionnaire (PHQ; Spitzer, Kroenke, \& Williams, 1999). The PHQ is a self-report measure keyed to DSM-IV criteria for mood, anxiety, eating, alcohol, and somatoform disorders in adults. Research using large samples of adults in medical settings supports the diagnostic validity and construct validity of the PHQ (Spitzer et al., 1999). 


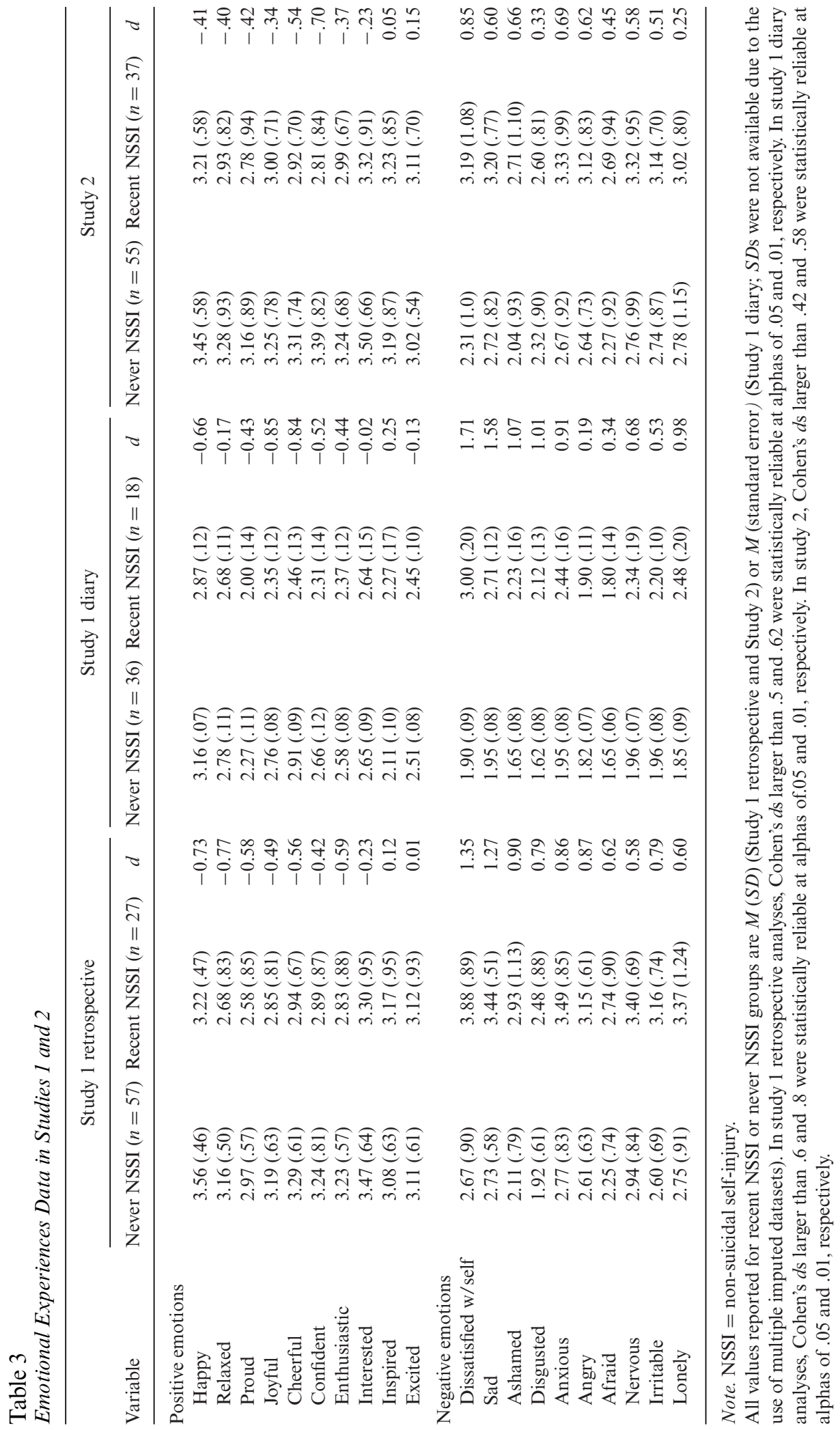


Results

Sociodemographics. Of the 92 participants included in these analyses, $59(64 \%)$ were female. Participants' ages ranged from 18 to 79 years, with an average of $30.3(S D=11.05)$. A substantial majority identified their ethnicity as Caucasian $(n=61,66.3 \%)$; other ethnic backgrounds were biracial/other $(n=11,12 \%)$, African-American $(n=7,7.6 \%)$, East Asian $(n=5,5.4 \%)$, Hispanic $(n=4,4.3 \%)$, South Asian $(n=3,3.3 \%)$, and Middle Eastern $(n=1,1.1 \%)$. The NSSI group was younger than those with no NSSI, $\mathrm{t}(85)=3.58, \mathrm{p}=.001$, $d=.68$.

Clinical characteristics. Complete clinical characteristics are presented in Table 1. Similar to the undergraduate sample, self-injurers exhibited more psychopathology than those without, including elevated levels of BPD symptoms, as assessed by the MSI-BPD, and elevated levels of depression, anxiety, and stress, as assessed by the DASS-21. Those with NSSI had a greater number of Axis I diagnoses, as assessed by the PHQ, than those without. Panic disorder, somatoform disorders, and bulimia nervosa were all significantly more likely among self-injurers; there were no significant group differences with respect to other diagnoses.

Among the participants with a history of NSSI, the median number of lifetime NSSI acts was 175. The mean number of methods of NSSI was $6.14(S D=3.13)$. In addition to cutting $(n=$ $28,75.7 \%)$, burning $(n=22,59.5 \%)$, and carving $(n=12,32.4 \%)$, other common methods of NSSI were biting, severe pinching, hairpulling, scratching, and interfering with wound healing. Complete data on NSSI history is presented in Table 2.

Specific emotions in NSSI. Of the 20 specific emotions, group differences were found for two positive emotions (cheerful and confident, lower in NSSI group), as well as eight negative emotions (sad, angry, afraid, ashamed, nervous, anxious, irritable, and dissatisfied with self, all higher in NSSI group). The largest effect was found for differences in the level of dissatisfaction with self. Detailed results for these specific emotions are reported in Table 3. After controlling for BPD symptoms, however, no specific emotions were significantly associated with NSSI history. Only self-dissatisfaction and cheer were robust to controlling for Axis I psychopathology (partial $r \mathrm{~s}=.26$ and -.37 , respectively). Similar to findings from study 1 , the inclusion of gender as a covariate did not change the magnitude or pattern of results.

\section{Discussion}

Previous research has highlighted the role of emotion in precipitating and motivating NSSI (Chapman \& Dixon-Gordon, 2007; Klonsky, 2007; Klonsky, 2009). However, this research has primarily relied on retrospective self-reports. The present research investigated daily emotional experience using a diary methodology, as well as retrospective versions of the diary measure to identify any areas of convergence or divergence between the two methodological approaches.

Findings in both samples and using both retrospective-report and diary measures indicated that individuals who self-injure show greater differences from noninjurers with respect to higher levels of negative emotions than to lower levels of positive emotions. In particular, dissatisfaction with self showed the greatest relationship to NSSI history of any specific positive or negative emotion; this was true in both undergraduate and community samples using both retrospective and daily diary methodologies. This finding was also robust to the inclusion of clinical covariates, including BPD symptoms and Axis I psychopathology, in most analyses. This finding is consistent with research suggesting that self-criticism is prominent in NSSI as compared to other, more indirect forms of self-harm (St. Germain \& Hooley, 2012). Self-directed negative emotional experiences, such as dissatisfaction with oneself, may make the direct injury inherent in NSSI more egosyntonic and less aversive to one's self-perception than it otherwise would be to individuals who view themselves more positively.

While the pattern of results was consistent across assessment methodologies and samples, there were some noteworthy differences between retrospective and diary findings in study 1 . In 
cases where larger effects were reported using retrospective measures, these differences may be due to floor effects in the diary assessments for emotions that are experienced less frequently (e.g., low endorsements of feeling afraid for both groups). Additionally, it may be the case that higher functioning self-injurers were more likely to agree to complete the diary, and would also be more similar to noninjurers than those who were unwilling to participate in the diary component of the study. Given that most effects were larger for emotions assessed using the diary, our results suggest that retrospective measures may not fully capture emotional experiences in NSSI.

Although this study has important strengths, such as the use of multiple assessment methodologies and samples, there are also several limitations that suggest future directions. First, participants were either undergraduate students or adults seeking out participation in online research. While the characteristics of NSSI in our samples (e.g., functions, methods, age of onset) were consistent with those of other populations of self-injurers, it is unclear to what extent study findings might differ in other populations, such as individuals receiving psychiatric care.

Second, our diary assessment methodology involved reporting daily emotions once per day; while this method may decrease recall bias evident in retrospective measures of emotion, which ask participants to report on emotions experienced days, weeks, or even months prior, our daily dairy assessment may still include some recall bias. Future studies could use methodologies such as ecological momentary assessment that more immediately capture emotional experiences.

Third, our research used a cross-sectional design, preventing inference of causal relationships between emotions and NSSI. For example, we are unable to determine whether selfdissatisfaction is a precipitant or consequence of NSSI, or both. Recent work using ecological momentary assessment technology suggests that NSSI is most often precipitated by feelings of anger towards the self; this suggests that NSSI may be related to not only trait self-dissatisfaction (as evidenced in our retrospective self-report findings) but also temporary state increases in self-directed negative emotions, which precipitate NSSI behaviors (Nock et al., 2009). Future research aimed at clarifying these relationships could be conducted through the use of laboratory paradigms that permit manipulations of self-dissatisfaction or other negative emotions prior to administration of NSSI proxies. In addition, longitudinal designs could establish temporal precedence of NSSI and associated emotional experiences.

\section{References}

American Psychiatric Association. (2000). Diagnostic and statistical manual of mental disorders (4th Ed., text revision). Washington, DC: Author.

Antony, M., Bieling, P., Cox, B., Enns, M., \& Swinson, R. (1998). Psychometric properties of the 42-item and 21-item versions of the depression anxiety stress scales in clinical groups and a community sample. Psychological Assessment, 10(2), 176-181. doi:10.1037/1040-3590.10.2.176

Buhrmester, M., Kwang, T., \& Gosling, S. D. (2011). Amazon's Mechanical Turk: A new source of inexpensive, yet high-quality, data? Perspectives on Psychological Science, 6(1), 3-5. doi:10.1177/1745691610393980

Chapman, A. L., \& Dixon-Gordon, K. L. (2007). Emotional antecedents and consequences of deliberate self-harm and suicide attempts. Suicide and Life-Threatening Behavior, 37(5), 543-552. doi:10.1521/suli.2007.37.5.543

Chapman, A. L., Gratz, K. L., \& Brown, M. Z. (2006). Solving the puzzle of deliberate self-harm: The experiential avoidance model. Behavior Research and Therapy, 44(3), 371-394. doi:10.1016/j.brat.2005.03.005

Chartrand, H., Sareen, J., Toews, M., \& Bolton, J. M. (2011). Suicide attempts versus nonsuicidal self-injury among individuals with anxiety disorders in a nationally representative sample. Depression and Anxiety, 29(3), 1-8. doi:10.1002/da.20882

Claes, L., Klonsky, E. D., Muehlenkamp, J., Kuppens, P., \& Vandereycken, W. (2010). The affect-regulation function of nonsuicidal self-injury in eating-disordered patients: Which affect states are regulated? Comprehensive Psychiatry, 51(4), 386-392. doi:10.1016/j.comppsych.2009.09.001

Cummings, J. A., Hayes, A. M., Cohen, L. H., Laurenceau, J., \& Saint, D. S. (2013). Interpersonal competence and daily stress generation in individuals with avoidant personality disorder symptoms. Journal of Social and Clinical Psychology, 32(2), 135-158. doi:10.1521.2Fjscp.2013.32.2.135 
First, M. B., Spitzer, R. L., Gibbon, M., \& Williams, J. B. (2002). Structured clinical interview for DSM-IVTR axis I disorders, research version, patient edition. New York, NY: Biometrics Research, New York State Psychiatric Institute.

Glenn, C. R., \& Klonsky, E. D. (2011). Prospective prediction of nonsuicidal self-injury: A 1-year longitudinal study in young adults. Behavior Therapy, 42, 751-762. doi:10.1016/j.beth.2011.04.005

Gratz, K. L., \& Tull, M. T. (2010). The relationship between emotion dysregulation and deliberate selfharm among inpatients with substance use disorders. Cognitive Therapy and Research, 34(6), 544-553. doi:10.1007/s10608-009-9268-4

Green, A. S., Rafaeli, E., Bolger, N., Shrout, P. E., \& Reis, H. T. (2006). Paper or plastic? Data equivalence in paper and electronic diaries. Psychological Methods, 11(1), 87-105. doi:10.1037/1082-989X.11.1.87

International Society for the Study of Self-Injury. (2007). About self-injury. Retrieved from http://www.isssweb.org/aboutnssi.php

Kamphuis, J. H., Ruyling, S. B., \& Reijntjes, A. H. (2007). Testing the emotion regulation hypothesis among self-injuring females: Evidence for differences across mood states. The Journal of Nervous and Mental Disease, 195(11), 912-8. doi:10.1097/NMD.0b013e3181593d89

Kleindienst, N., Bohus, M., Ludäscher, P., Limberger, M. F., Kuenkele, K., Ebner-Priemer, U. W., .. . Schmahl, C. (2008). Motives for nonsuicidal self-injury among women with borderline personality disorder. The Journal of Nervous and Mental Disease, 196(3), 230-6. doi:10.1097/NMD.0b013e3181663026

Klonsky, E. D. (2007). The functions of deliberate self-injury: A review of the evidence. Clinical Psychology Review, 27(2), 226-39. doi:10.1016/j.cpr.2006.08.002

Klonsky, E. D. (2009). The functions of self-injury in young adults who cut themselves: Clarifying the evidence for affect-regulation. Psychiatry Research, 166(2-3), 260-268. doi:10.1016/j.psychres.2008.02.008

Klonsky, E. D. (2011). Non-suicidal self-injury in United States adults: Prevalence, sociodemographics, topography and functions. Psychological Medicine, 41(9), 1981-1986. doi:10.1017/S0033291710002497

Klonsky, E.D., May, A., \& Glenn, C.R. (2013). The relationship between non-suicidal self-injury and attempted suicide: Converging evidence from four samples. Journal of Abnormal Psychology, 122(1), 231-237. doi:10.1037/a0030278

Klonsky, E. D., \& Olino, T. M. (2008). Identifying clinically distinct subgroups of self-injurers among young adults: A latent class analysis. Journal of Consulting and Clinical Psychology, 76(1), 22-27. doi:10.1037/0022-006X.76.1.22

Klonsky, E. D., Oltmanns, T. F., \& Turkheimer, E. (2003). Deliberate self-harm in a nonclinical population: Prevalence and psychological correlates. American Journal of Psychiatry, 160(8), 1501-1508. doi:10.1176/appi.ajp.160.8.1501

Laye-Gindhu, A., \& Schonert-Reichl, K. A. (2005). Nonsuicidal self-harm among community adolescents: Understanding the "whats" and "whys" of self-harm. Journal of Youth and Adolescence, 34(5), 447-457. doi:10.1007/s10964-005-7262-Z

Lovibond, P. F., \& Lovibond, S. H. (1995). The structure of negative emotional states: Comparison of the depression anxiety stress scales (DASS) with the beck depression and anxiety inventories. Behaviour Research and Therapy, 33(3), 335-343. doi:10.1016/0005-7967(94)00075-U

Lovibond, S. H., \& Lovibond, P. F. (1995). Manual for the Depression Anxiety Stress Scales. . (2nd Edition). Sydney: Psychology Foundation

Moore, T. M., Elkins, S. R., McNulty, J. K., Kivisto, A. J., \& Handsel, V. A. (2011). Alcohol use and intimate partner violence perpetration among college students: Assessing the temporal association using electronic diary technology. Psychology of Violence, 1(4), 315-328. doi:10.1037/a0025077

Nock, M. K., Joiner, T. E., Gordon, K. H., Lloyd-Richardson, E., \& Prinstein, M. J. (2006). Non-suicidal selfinjury among adolescents: Diagnostic correlates and relation to suicide attempts. Psychiatry Research, 144(1), 65-72. doi:10.1016/j.psychres.2006.05.010

Nock, M. K., \& Prinstein, M. J. (2005). Contextual features and behavioral functions of selfmutilation among adolescents. Journal of Abnormal Psychology, 114(1), 140-146. doi:10.1037/0021843X.114.1.140

Nock, M. K., Prinstein, M. J., \& Sterba, S. K. (2009). Revealing the form and function of self-injurious thoughts and behaviors: A real-time ecological assessment study among adolescents and young adults. Journal of Abnormal Psychology, 118(4), 816-827. doi:10.1037/a0016948

Pfohl, B., Blum, N. S., \& Zimmerman, M. (1997). Structured interview for DSM-IV personality: SIDP-IV. Washington, DC: American Psychiatric Press.

Rogers, R. (2001). Handbook of diagnostic and structured interviewing. New York, NY: Guilford Press. 
Selby, E. A., Bender, T. W., Gordon, K. H., Nock, M. K., \& Joiner, T. E. (2012). Non-suicidal self-injury (NSSI) disorder: A preliminary study. Personality Disorders, 3(2), 167-75. doi:10.1037/a0024405

Spitzer, R. L., Kroenke, K., \& Williams, J. B. M. (1999). Validation and utility of a self-report version of the PRIME-MD: The PHQ primary care study. Journal of the American Medical Association, 282, 1737-1744. doi:10.1001/jama.282.18.1737

St Germain, S. A., \& Hooley, J. M. (2012). Direct and indirect forms of non-suicidal self-injury: Evidence for a distinction. Psychiatry Research, 197(1-2), 78-84. doi:10.1016/j.psychres.2011.12.050

Zanarini, M. C., Skodol, A. E., Bender, D., Dolan, R., Sanislow, C., Schaefer, E., ... Gunderson, J. G. (2000). The collaborative longitudinal personality disorders study: Reliability of Axis I and II diagnoses. Journal of Personality Disorders, 14(4), 291-299.

Zanarini, M. C., Vujanovic, A. A., Parachini, E. A., Boulanger, J. L., Frankenburg, F. R., \& Hennen, J. (2003). A screening measure for BPD: The Mclean screening instrument for borderline personality disorder (MSI-BPD). Journal of Personality Disorders, 17(6), 568-573. doi:10.1521/pedi.17.6.568.25355

Zlotnick, C., Shea, M. T., Pearlstein, T., Simpson, E., Costello, E., \& Begin, A. (1996). The relationship between dissociative symptoms, alexithymia, impulsivity, sexual abuse, and self-mutilation. Comprehensive Psychiatry, 37(1), 12-16. doi:10.1016/S0010-440X(96)90044-9 